\title{
Molecular phylogeny and evolution of tropical vegetal genus Coccinia (Cucurbitaceae) using chloroplast barcode markers and its implications for speciation patterns
}

\author{
Mala P., Payel D., Sunita S.*
}

School of Biotechnology and Bioinformatics, D. Y. Patil University, Navi- Mumbai, India.

Received: 2017-10-24; Accepted: 2017-10-26

\begin{abstract}
Evaluation of molecular phylogeny employing barcode markers for a population analysis has revealed the association between character evolution and diversification. The present research study endeavors to estimate the molecular phylogeny and evolution amongst 30 geographically distant land races of Coccinia grandis using chloroplast barcode markers. On the basis of sequences of C. grandis landraces and sister genera's of Cucurbits; phylogeny and evolutionary distances were estimated using bootstrap method of UPGMA and ML with MEGA and DAMBE software. The $r b c \mathrm{~L}$ appear to be evolving marginally amongst land races studied, while matK loci exhibited several parsimonically insignificant variations. The ycf locus resolved efficiently the phylogeny at both intraspecies and intergeneric levels. The values of Tajima's relative rate test, Nei's genetic distance and gamma distribution, within $C$. grandis accessions and among Cucurbits denoted plausibility of occurrences of random population expansion, slow ontogenic transitions and high consanguinity percentage among core barcode regions. The present study thus lays a platform for researchers working with vegetatively propagated plants to plan strategies for exchange of varieties, their maintenance, utilization and enhancement for further crop development and propagation.
\end{abstract}

Keywords: Coccinia grandis, character evolution, diversification, phylogeny, genetic distance

\section{Introduction}

Phylogenetic relationship is a collective upshot of existing ecological exchanges, environmental shifts, and evolutionary chronicles. It often links community ecology with biogeography and trait evolution (Wang et al., 2009b). The perplexing diversity, plasticity and phylogenetic consanguinity have prompted several plant biotechnologists to resolve finer-scale phylogenetic queries and spatial distribution of genealogical lineages to read the influence of historical processes on the evolution of populations and species, employing some Single loci Amplicon Profiling (SLAP) markers, also known as 'DNA Barcode's (Zhang et al., 2012). The 'gene tree' generated employing one or more barcode markers not only date the evolutionary history of a phylum but are also employed to predict speciation and/ or evolutionized patterns (Duminil \& Michele 2009). For inferring the phylogeny, earlier use of a single plastome or nucleome gene, (Chase et al., 1993) and later assorted combinations of four plastid coding genes $(r p o B, r p o C 1, r b c L$ and matK) and four intergenic spacers (atpF-atpH, $p s b K-p s b I$, $\operatorname{trn} S^{G C U}-\operatorname{trn} G^{U C C}$ and $\operatorname{trn} H-p s b A$ ) along nuclear genes have become fundamental for evolutionary botanists (Davis et al., 2014).

\footnotetext{
*Corresponding Author:

Dr. Sunita Singh,

Associate Professor and Head,

Biotechnology Section, SBB-DYPUNM,

Convener, Research Advisory Committee

and Institutional Bio-safety Committee,

D. Y. Patil University Belapur, Navi Mumbai,

Maharashtra-400614, India.

E-mail: dr.sunitads@gmail.com
}

Cucurbitaceae, today's economically vital group, encompasses a vast variety of species that provide human with edible products and beneficial fibers (Bisognin, 2002). Plants of this family are very similar with respect to leaf and stem structures, but they have high genetic diversity for fruit shape and other fruit characteristics. It consists of about 119 genera with 825 species including Coccinia grandis L. voigt. (Ivy gourd, Scarlet gourd), which is a tropical, dioecious and feral vegetal vine (Muniappan et al., 2009). The plant is indigenous to central East Africa but has naturalized in Asia, Australia, Pacific Islands and Caribbean Islands (Holstein \& Renner 2011). In addition to its dietary uses, this ethnic tribal plant has numerous therapeutic virtues viz. anti-diabetic, anti-ulcer, anti-inflammatory, anti-oxidant and anticancer properties (Vadivu et al., 2008; Deokate \& Khadabadi 2012). This vegetatively propagated crop encompasses a huge range of phylogenetic, morphological and ecological diversity. The genus has been the focal point of numerous ecological studies on the sexual system (Shaina and Beevy, 2015) and the interactions with various insects in the community (Muniappan, 2009). The plant being clonally propagated, under diverse ecologies and the convoluted mix of selection pressures acting on the harvested branch and other clonal propagules, may 
result in complex and diverse evolutionary trajectories under domestication (Mckey et al., 2010). Domestication is thus an evolutionary process through which domesticated plants become morpho-physiologically divergent from their wild ancestors (Harlan 1992). Understanding the evolutionary history and domestication process of the species therefore is imperative for better exploitation of genetic diversity and cultivar development.

The present study uses barcode markers to evaluate nucleotide sequence variation in chloroplast and predict speciation pattern and community phylogenetics among land races of $C$. grandis. The assessment of variance in core genomic region of Ivy gourd will shed information for devising and executing effective breeding programs for local crop improvement and effective utilization of this vegetal species.

\section{Materials and Methods}

\section{Plant Material:}

The genotypes mentioned in our previous study (Singh \& Parab, 2015) were employed in this study for molecular typing of C. grandis.

\section{Genotyping:}

The genomic DNA was extracted using $m C T A B$ method described by Payel et al., (2015). The DNA was amplified using locus specific primer as described by Kress et al., (2007) at three separate loci corresponding to the commonly used chloroplast barcode DNA markers (1) 455 bp of the ribulose-bisphosphate/carboxylase Large-subunit gene $(r b c \mathrm{~L}),(2)$ approximately $680 \mathrm{bp}$ of the maturase-K gene (matK) (3) 300 bp of the unknown chloroplast gene $(y c 5)$.

\section{Diversity Analysis:}

For diversity and phylogeny analysis, sequences of different Coccinia species and some genera among Cucurbits were retrieved from GenBank, while for Coccinia grandis only original sequence data generated in present study were utilized. The sequences of each gene were aligned using ClustalO (EMBLEBI) with default parameters. Aligned sequences were manually inspected and adjusted using DNA Sequencher (ver. 5.2.4 Gene Codes Corp.) and only the core regions i.e. the sequence completely aligned and of similar length, were used for further analyses. A phylogram was constructed using bootstrap method of UPGMA (Unweighted Pair Group Method with Arithmetic Average), ML (Maximum Likelihood) using programs MEGA 6 (Molecular Evolutionary Genetic Analysis, ver. 6) (Tamura et al., 2013) and DAMBE (Data analysis in Molecular Biology and Evolution, ver. 5.5.16) (Xia 2013). Also the correlation of Ivy gourd at intra/ inter genus was evaluated using Neighbor Joining (NJ) method (data not shown). Species discriminations and evolutionary adaptations amongst this vegetal crop landrace were deduced by PWG- distance, Tajima's relative rate test (TRRT), Gamma distribution (GD) and Nei's genetic distances (NGD). The sequence data generated from this study has been communicated to GenBank (Table 1).

Table 1: Sequences of C. grandis deposited with GenBank

\begin{tabular}{lccc}
\hline Sr. No & C. grandis Land Variety & cp- SLAP Locus & Accession No \\
\hline 1. & Coccinia grandis cultivar MS15K & tRNA-Gln (tmQ) and $t$ RNA-Ser(tmS2) & KP982899 \\
2. & (CgVM15)- ycf 1 primer & $y c f 5$ & KR049232 \\
3. & CgVM5 & $y c f 5$ & KR049233 \\
4. & CgVM13 & $y c f 5$ & KR049234 \\
5. & CgVM6 & matK & KT356186 \\
6. & CgVM4 & matK & KT356187 \\
7. & CgVM6 & matK & KT356188 \\
8. & CgVM16 & matK & KT356189 \\
CgVM10 & CgVM10 & $r b c L$ & KU165658 \\
9. & CgVM11 & $r b c L$ & KU165659 \\
10. & CgVM12 & $r b c L$ & KU165660 \\
11. & CgVM13 & $r b c L$ & KU165661 \\
12. & CgVM14 & $r b c L$ & KU165662 \\
13. & CgVM15 & $r b c L$ & KU165663 \\
14. & CgVMP27 & $r b c L$ & KU165664 \\
15. & CgVMS22 & $r b c L$ & KU165665 \\
\hline
\end{tabular}

\section{Results}

Phylogram analyses in present study involved evaluation of genetic distances and correlating evolution at all three levels (intraspecies, interspecies and intergeneric) within C. grandis landraces. It also checked the predisposition of each chloroplast DNA marker to be used as "barcode" for C. grandis. Employing different DNA markers the intraspecies and interspecies divergence was observed to vary from $0.0038-2.5$ and $0.0065-0.38$ respectively, while intergenus diversity was evaluated to range from 0.52 to 1.81 (Table 2). The average nucleotide substitution rate for all the chloroplast markers was observed in the range of 0.7 to 0.8 while the tree length were observed to be in proportion to the rate of substitution per site. 
Using $r b c \mathrm{~L}$ marker the number of segregating sites and the average number of nucleotide differences was estimated from PWG-distances, which emphasize a coterminous relationship within Coccinia spp. and other Cucurbitaceae genus. The distribution of fitness effects of new mutations was estimated using gamma distributions shape parameter (0.26 - 5.4, Table 2). The phylogram generated using $r b c \mathrm{~L}$ loci specific marker, exhibited homogenous distributions of C.grandis landraces, as they constellated in one phylode, except CgMP25 (Table 2, Figure 1). Upon intergenus comparisons a complete homology of the sequences for $r b c \mathrm{~L}$ loci was revealed (Figure 2). On contrary the matK loci revealed short sequence "types" and ontogenic transmutations, leading to distribution of landraces in different clades (Figure 3). However, these variations were scored to be parsimonically nonsignificant. Intra- and inter- species comparisons revealed a high correlation among the Coccinia spp. accessions and $\mathrm{CgVM} 10$ and CGVM16 as they were clustered in one phylode (Figure 3), while land races $\mathrm{CgVM} 4$ and $\mathrm{CgVM} 6$ were placed in another clade. Thus the former land races revealed high kinship with other species of Coccinia and may be a recent outcome of geological timescale. Intergenus analysis illustrated a close relatedness amongst the Coccinia spp. accessions and other member of Cucurbitaceae, while land races $\mathrm{CgVM} 4$ and CgVM6 were observed to be placed in another clade, as compared to out-grouped Echinopepon insularis (Figure 4). The transmutation detected in this locus appears to be a reflection of differential rates of transition/ transversion mutations in different sectors of the mat $\mathrm{K}$ gene (Liang \& Hilu 1996).

The intraspecies phylogenetic analysis using $y c f 5$ exhibited clustering of land races in phylodes with respect to observed sequence variation and their geological transmutations; however, none of these variations scored were parsimonically informative (Table 2).

Table 2: Genetic distances calculated from different SLAP chloroplastid loci

\begin{tabular}{llllllllll}
\hline SLAP loci & CL & PS $^{*}$ & SR & TRT $^{*}$ & GP $^{*}$ & NGD $^{\phi}$ & ISD $^{* \#}$ & InD $^{* \otimes}$ & IGD $^{* \boldsymbol{\Phi}}$ \\
\hline matk & $\mathbf{6 8 0}$ & $\mathbf{5 2}$ & 0.72 & 0.47 & 4.21 & $0.021 \pm 0.01$ & 0.6500 & 0.0065 & 0.52 \\
rbcL & 455 & 8 & 0.7 & 0.033 & 4.318 & $0.167 \pm 0.08$ & 0.0038 & 0.038 & 0.64 \\
$y c f-5$ & 300 & 43 & $\mathbf{0 . 8}$ & 1.5 & 0.26 & $0.033 \pm 0.017$ & $\mathbf{2 . 5 0 0}$ & ----- & $\mathbf{1 . 8 1}$ \\
\hline
\end{tabular}

Note: $(1) *$ : Evaluated using Mega 6.0 software, (2) $\phi$ : estimated using DAMBE 5.5.16 software, (3) \#: estimation based on only Coccinia grandis land races (4) $\uparrow$ : estimation based on Coccinia spp. and related Cucurbits (5) $\otimes$ estimation based on Coccinia spps.(6) CL: contig length (7) PS: Parsimony site (8) SR: Substitution rate (9) TRT: Tajimas relative rate test (10) GP: Gamma distribution shape parameter (11) NGD: Nei's genetic distance (12) ISD: Intra-specific divergence (13) InD: Inter species divergence (14) IGD: Inter genus divergence

Intergenus analysis demonstrated dissimilarity and remote geno-relations amongst the $C$. grandis land races and other members of Cucurbitaceae (Figure 5). The C. grandis landraces were clustered together in one phylode except for CgVM5, while the rest of the Cucurbits were distributed with respect to their variability in the residual four clades. Thus in present study higher variation in $y c f 5$ allowed recovery of several topologies (data not shown) in Coccinia and other Cucurbits for the first time.

\section{Discussion}

Plant taxonomy, the science of cataloging plant systems on basis of communal traits, has always been vital, as it facilitates monitoring the biological effects of global climate alterations and attempt to conserve species diversity with increasing habitat destruction. Chloroplast DNA based barcode markers enables rapid and accurate identification of plant species; however, none of the available loci are highly informative across all plant species. The present research endeavours to divulge the evolutionary and speciation patterns in geologically separated landraces of $C$. grandis executing three barcode DNA markers from chloroplast region.

Phylogenetic analysis employing markers like $r b c \mathrm{~L}$ exhibited high genetic relatedness and thus may not be suitable for species identification among land races analyzed herein. Thus on the basis of $r b c \mathrm{~L}$ sequence variations among Coccinia and other Cucurbit spp. in present study appears to demonstrate chloroplast homoplasy, reticulate speciation pattern. Our results are consistent with the previous studies of Small et al., (1998) who have reported reduced difference at lower taxonomic strata in phylogenies as an outcome of functional constraints of chloroplast coding genes. Homoplasy in $r b c \mathrm{~L}$ sequences can also be attributed to polyploid chromosomes in C. grandis, as multiple allo-/ autopolyploid species are observed to share a common parent species and have identical plastid sequences (Hollingsworth et al., 2011). Furthermore, presence of cellular symbionts, interspecific gene introgression or reticulate speciation pattern transpires incomplete lineage sorting and allocation of similar sequences between species (Min \& Hickey 2007). Rapidly evolving sites in the genome enhance the chance of generating synapomorphies without being obscured by multiple hits, simply by expanding the information at nucleotide level. Our predictions for homoplasy among Coccinia spp. are also supported by previous studies of Borsch et al., (2003) that have demonstrated prefered tendency of co-emergence of certain chloroplast $r b c \mathrm{~L}$ loci and/ or presence of repetitive nucleotides thereby favoring protein structural stability. 


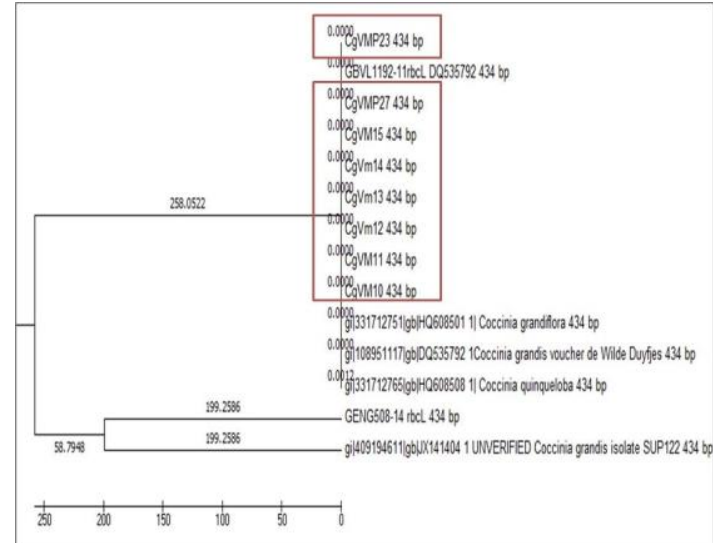

Figure 1. Phylogram and ancestry reconstruction within Coccinia species generated by UPGMA based bootstrapping employing Chloroplastid rbcL2 loci. Numbers on branches refer to bootstrap support $>$ $75 \%$. The tree is rooted on C. grandis isolate SUP122.

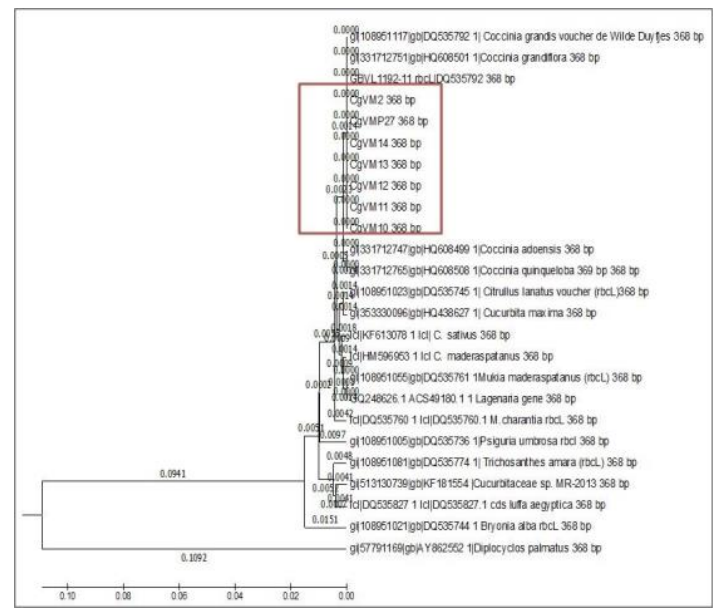

Figure 2. Phylogram and ancestry reconstruction within Cucurbitaceae generated by UPGMA based bootstrapping employing Chloroplastid rbcL2 loci. Numbers on branches refer to bootstrap support $>$ $85 \%$. The tree is rooted on Bryonia alba and $D$. palmatus was used as out group.

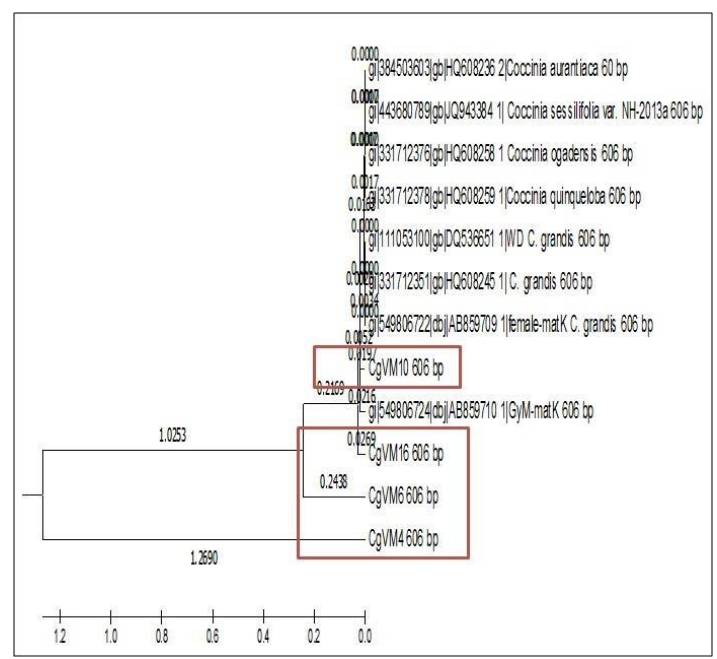

Figure 3. Phylogram and ancestry reconstruction within Coccinia species generated by UPGMA based bootstrapping employing Chloroplastid matK loci.
Numbers on branches refer to bootstrap support $>80 \%$. The tree is rooted on $\mathrm{CgVM} 4$.

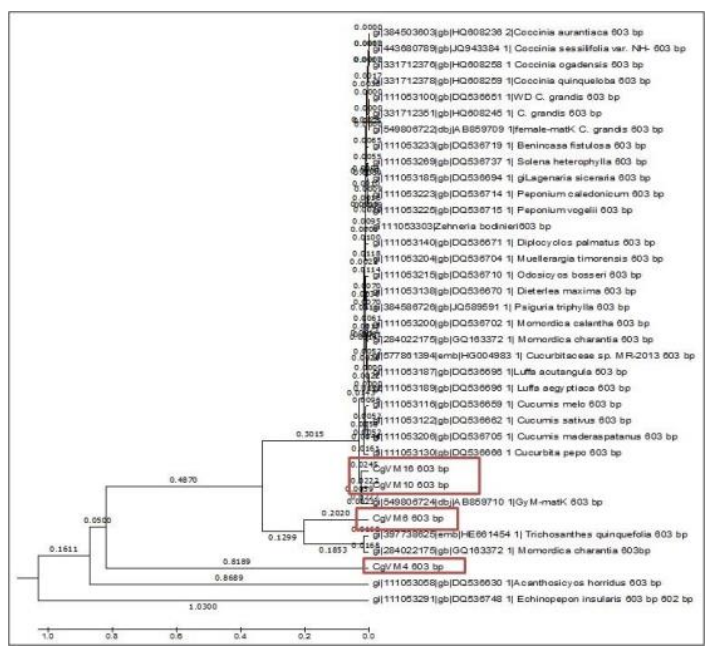

Figure 4. Phylogram and ancestry reconstruction within Cucurbitaceae generated by UPGMA based bootstrapping employing Chloroplastid matK loci. Numbers on branches refer to bootstrap support $>80 \%$. The tree is rooted on CgVM4 and $A$. horridus. Echinopepon insularis was used as out group.

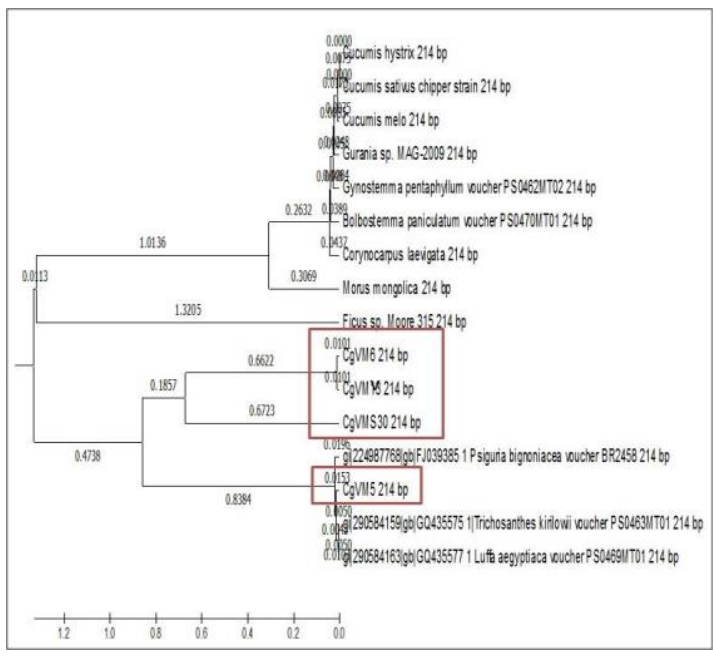

Figure 5. Phylogram and ancestry reconstruction within Cucurbitaceae generated by UPGMA based bootstrapping employing chloroplastid ycf5 loci. Numbers on branches refer to bootstrap support $>$ $80 \%$. The Cucumis bystrix was used as out group.

The matK exhibited multiple sequence variations among Coccinia spp. and thus may be proficiently used for inter-species variance analysis. In the present study matK gene appears to be under far less selectional constriction as is evident from the considerably higher rate of nonsynonymous substitution that is up to 22 times that of other genes and about five-fold that of $r b c \mathrm{~L}$. A similar finding had been previously reported by Liang and Hilu (1996). Furthermore, Barthet and Hilu (2007) had reported that rate of substitution in mat $\mathrm{K}$ is three times more at the nucleotide level and is six times high at the amino acid level than that of $r b c \mathrm{~L}$. 
In present study matK barcode marker demonstrated diversity for individual clades, subclades with better resolution within the family Cucurbitaceae than that obtained from $r b c \mathrm{~L}$. Thus, the matK locus examined herein was perceived to be a putative paralog and the species appear to demonstrate paraphyletic evolution, as reported in previous studies by Reddy (2009) for several accessions of Cucurbitaceae and thus possibly can be used for resolving the supra-generic groupings of selective members.

Similarly, $y c 5$, required for heme attachment to chloroplast c-type cytochromes (Tsuruya et al., 2006), was recorded as a good barcode marker at both intra-species and intergenus level. Previous studies have reported use of $y c f 5$ in combination with different plastid regions for phylogeny analysis viz. diversity among species of Allium (Anvarkhah et al., 2013), Amazonian trees (Gonzalez et al., 2009), medicinally significant angiosperms, gymnosperms and fungi (Chen et al., 2010), analysis of flora in biodiversity hotspots in South Africa (Lahaye et al., 2008) and intra/ inter species divergence in family Rutaceae (Kun Luo et al., 2010). In present study the phylogenetic analyses conducted employing ycf5 resulted in congruent trees revealing high kinship among the genus Coccinia with respect to the outgroup genera's chosen. In this gradually evolving region subtle variation in secondary structures, multiple-hit sites and intra-molecular recombinational sequences were also detected for first time in C. grandis by our study (data not shown).

The flow of evolution among C. grandis was statistically validated by calculating genetic distances by TRT, GD and NGD (Table 2). The values of TRT and GD indicated that there are several parsimonically significant sites with mat $K$ and $y c f 5$ that are evolving at a higher rate, while the contig of other regions evaluated herein are evolving at a very slow rate. These statistics based estimations probe diversity within or between ecological populations, to examine the genetic variation in related species, or to determine evolutionary relationships. The values of all the genetic distances with respect to $r b c \mathrm{~L}$ loci demonstrated that Coccinia is a gradually evolving vegetal crop and these chloroplast regions exhibited phyletic gradualism. On the contrary the matK and ycf5 loci indicated that the land races appeared to be descendants of balancing selection wherein the random population expansion and / or modest ontogenic transitions are occurring in some core regions of $C$. grandis so as to get acclimatized in varying ecological environments. The geographic distribution of cytonuclear discordance often reflects genetic introgression patterns across a species of a geographical range.

\section{Conclusion}

The variations in methods of domestication and propagation along with geo-edaphic conditions inculcate molecular evolution in both chloroplast genomes. C. grandis has withstood six alliterative biome shifts, thus phylogeny reconstruction with more accurate disposition of Coccinia sp. at intraspecies and inter-genus has been the quest of present research study. Phylogenetic analysis employing the three-chloroplast barcode DNA enabled us to discern between gene pool division emerging from primeval phylogeographic splits to recent ecological divergence, wherein a decrease in gene pool diversity were recovered. We therefore put forward on the basis of sequence variations recorded in our samples of C. grandis varieties, these species are being enrouted towards parallel and/ or divergent evolution and different alleles appeared to be assorted under random speciation.

\section{Acknowledgements}

We are thankful to D. Y. Patil University for infrastructural support. We are also thankful to Late Dr. Madhusudan Samant, Assistant Professor, School of Biotechnology and Bioinformatics, D. Y. Patil University (SBB, DYPU) and Mr. Pramodkumar G., Assistant Professor, SBB- DYPU for providing us technical guidance for online gene identification software's and sequence annotations.

\section{References}

1. Anvarkhah S, Hosseini M, Mohassel M, Panah A, and Hashemi H. "Identification of three species of genus Allium using DNA barcoding." Intl J Agri Crop Sci, 5.11 (2013): 1195-1203. http://ijagcs.com/wpcontent/uploads/2013/05/1195-1203.pdf

2. Barthet $\mathrm{M}$ and Hilu $\mathrm{K}$. "Expression of matk: functional and evolutionary implications." A. J. Bot 4.(2007):1402-1412.

http://www.amjbot.org/content/94/8/1402.long

3. Bisognin D. "Origin and evaluation of cultivated cucurbits." Ciencia Rral. 32.5 (2002): 715-723. https://www.academia.edu/31754521/Origin_and_ evolution_of_cultivated_cucurbits

4. Borsch T, Hilu KW, Quandt W, Wilde V, Neinhuis $\mathrm{C}$, and Barthlott W. "Noncoding plastid trn'T-trnF sequences reveal a well resolved phylogeny of basal angiosperms." J Evol Biol. 16(2003): 558-576. https://www.ncbi.nlm.nih.gov/pubmed/14632220

5. Chase M, Soltis D, Olmstead R, Morgan D, Les D, Mishler B, Duvall M, Price R, et al. "Phylogenetics of seed plants: an analysis of nucleotide sequences from the plastid gene rbcL" Ann Missouri Bot Gard, 80(1993):528-580.

http://loco.biosci.arizona.edu/PDG/pdfs/Chase19 93.pdf

6. Chase MW and Fay MF. "Barcoding of plants and fungi." Science, $325 \quad$ (2009):682-683. 
http://science.sciencemag.org/content/325/5941/6 82

7. Chen S, Yao H, Han J, Liu C, Song J, Shi L, Zhu Y, $\mathrm{Ma} \mathrm{X}, \mathrm{Gao}$ et al. "Validation of the ITS2 region as a novel DNA barcode for identifying medicinal plant species." PLoS ONE. 5 (2010). e8613. http://journals.plos.org/plosone/article?id=10.1371 /journal.pone.0008613

8. Davis $\mathrm{C}, \mathrm{Xi} \mathrm{Z}$ and Mathews S. "Plastid phylogenomics and green plant phylogeny." $B M C$ Biology. $\quad 12.11 \quad$ (2014). http://www.biomedcentral.com/1741-7007/12/11

9. Deokate U and Khadabadi S. "Pharmacology and photochemistry of Coccinia indica." Pharmacophore. 3.3 (2012): 179-185.

http://www.pharmacophorejournal.com/MayJune2012-article3.pdf

10. Duminil J and Michele M. "Plant species delimitation: a comparison of morphological and molecular markers." Plant Biosystematics. 143 (2009): 528-542.

http://ebe.ulb.ac.be/ebe/

Duminil_files/Duminil $\% 20 \% 26 \% 20$ al. $\% 202009 \% 2$ OPB.pdf

11. Gonzalez M, Baralato C, Moris S, Petronelli P, Reira $\mathrm{B}$, Thebaund $\mathrm{C}$ and Chave J. "Identification of Amazonian trees with DNA barcodes." PLoS ONE, 4 (2009): e7483.

http:/ / journals.plos.org/plosone/article?id=10.1371 /journal.pone.0007483

12. Harlan JR. "Origins and processes of domestication. In: Chapman GP (ed) Grass evolution and domestication." Cambridge University Press, Cambridge, (1992): 159-175.

http://pdf.usaid.gov/pdf_docs/pnacf852.pdf

13. Hollingsworth P, Graham S and Little D. "Choosing and using a plant DNA Barcode." PLOS ONE. 6.5 (2011): e19254. doi:10.1371/journal.pone.0019254. http://www.ncbi.nlm.nih.gov/pmc/articles/PMC 3261039/ pdf/PhytoKeys-007-027.pdf

14. Holstein N and Renner S. "Coccinia intermedia - a new Cucurbitaceae species from West Africa." Phytokeys. 7 (2011): 27-36.

https://phytokeys.pensoft.net/article/1396/downlo ad/pdf/

15. Kress W and Erickson D. "A two-locus global DNA barcode for land plants: The coding rbcL gene complements the non-coding trnH-psbA spacer region." PLOS ONE, (2007) doi.10.1371/journal.pone.0000508.

16. Kun Luo Chen S, Chen K, Song J, Yao H, Ma X, Zhu Y, Pang X, Yu H, Li X and Liu, Z. "Assessment of candidate plant DNA barcodes using the Rutaceae family." Science China, Life Sci. 53 6 (2010): 701-708.

http://journals.plos.org/plosone/article?id=10.1371 /journal.pone.0000508.
17. Lahaye R, Van der Bank M, Bogarin D, Warner J, Pupulin F, Gigot G, Maurin O, Duthoit S, Barraclough, T and Savolainen V. "DNA barcoding the floras of biodiversity hot spot." PNAS, USA, 105 (2008): 2923-2928.

https://www.ncbi.nlm.nih.gov/pmc/articles/PMC2 268561/

18. Liang $\mathrm{H}$ and Hilu $\mathrm{K}$. "Application of the matK gene sequences to grass systematic." Canadian J. Bot. 74 (1996): 125-134.

http://www.nrcresearchpress.com/doi/abs/10.1139 /b96-017\#.WXcNe1H-vIU

19. Mckey D, Elias M, Pujol B and Duputie A. "The evolutionary ecology of clonally propagated domesticated plants." New Phytol. 186 (2010): 318 332

https://www.ncbi.nlm.nih.gov/pubmed/20202131

20. Min X and Hickey D. "DNA barcodes provide a quick preview of mitochondrial genome composition." PLOS ONE. $2.3 \quad$ (2007): e325. http://journals.plos.org/plosone/article?id=10.1371 /journal. pone.0000325

21. Muniappan R, Reddy G, and Raman A. "Coccinia grandis (L.) Voigt. (Cucurbitaceae) Biological Control of Tropical Weeds used Arthropods. Cambridge University Press. (2009): 175-182.

http://assets.cambridge.org/97805218/77916/front matter/9780521877916_frontmatter.pdf

22. Nei M. "Genetic distance and molecular phylogeny." Columbia University Press, New York (2000): 193-223. http://nsgl.gso.uri.edu/washu/washub87001/washu b87001_part6.pdf

23. Payel D, Mala $\mathrm{P}$, and Sunita S. "Inter genus variation analysis in few members of Cucurbitaceae using ISSR markers." Biotechnology and Bioequipment. 29.5 (2015): 882-886.

DOI: $10.1080 / 13102818.2015 .1052760$.

24. Reddy U. "A phylogenetic analysis of the Cucurbitaceae: evidences from matk nucleotide sequences." International Journal of Bioinformatics Research. 1.2 (2009): 47-53.

https://bioinfopublication.org/viewhtml.php?artid= BIA0001340

25. Reddy U. "Cladistic analysis of few members of Cucurbitaceae using rbcL nucleotide and amino acid sequences." International Journal of Bioinformatics Research, 1.2 (2009): 58-64.

https://bioinfopublication.org/viewhtml.php?artid= BIA0001342

26. Shaina $\mathrm{T}$ and Beevy S. "Reproductive biology of Coccinia grandis (L) Voigt. a dioecious vegetatively propagated Cucurbit: evidence for facultative apomixes." Int J Plant Reprod Biol. 7.1 (2015): 67-77. http://ijprb.com/vol\%2007\%20(1)/pdf\%2007.pdf

27. Singh S and Parab M. "Fingerprinting intra-specific diversity among Coccinia grandis landraces." Int. J. Recent Sci. Res. 6.3 (2015): 3025-3032. 
http://www.recentscientific.com/sites/default/files /2063.pdf

28. Small R, Ryburn J, Cronn R, Seelanan T and Wendel $\mathrm{J}$. "The tortoise and the hare: choosing between noncoding plastome and nuclear adh sequences for phylogenetic reconstruction in a recently diverged plant group." Am. J. Bot. 85 (1998): 1301-1315. https://www.ncbi.nlm.nih.gov/pubmed/21685016

29. Tajima F. "Statistical method for testing the neutral mutation hypothesis by DNA polymorphism." Genetics. 123.3 (1989): 585- 595.

https://www.ncbi.nlm.nih.gov/pmc/articles/PMC1 203831

30. Tamura K, Stecher G, Peterson D, Filipski A and Kimar S. "MEGA6: Molecular Evolutionary Genetics Analysis version 6.0." Molecular Biology \& Evolution. (2013). doi: 10.1093/molbev/mst197. https://www.ncbi.nlm.nih.gov/pmc/articles/PMC3 840312

31. Tsuruya K, Suzuki M, Plader W, Sugita C and Sugita M. "Chloroplast transformation reveals that tobacco $y c f 5$ is involved in photosynthesis." Acta Physiol. Plant 28 (2006): 365- 371

https://link.springer.com/article/10.1007\%2Fs1173 8-006-0033-y

32. Vadivu R, Krithika A, Biplab C, Dedeepya P, Shoeb and Lakshmi K. "Evaluation of hepatoprotective activity of the fruits of Coccinia grandis Linn. Int. J. Health Res. 3 (2008): 163-168. https://www.ajol.info/index.php/ijhr/article/view/ 55366

33. Wang Z, Brown J, Tang $Z$ and Fang J. "Temperature dependence, spatial scale, and tree species diversity in eastern Asia and North America." PNAS, USA. 106.32 (2009b): 1338813392.

http://www.pnas.org/content/106/32/13388.

34. Xia X. "DAMBE 5: A comprehensive software package for data analysis in molecular biology and evolution.” Mol. Biol. \& Evol. 30 (2013): 1720-1728. https://www.ncbi.nlm.nih.gov/pmc/articles/PMC3 684854

35. Zhang, C, Pratap, A, Natarajan S, Pugalendhi L, Shinji, K, Sassa H, Senthil $\mathrm{N}$ and Kobal T. "Evaluation of Morphological and Molecular Diversity among South Asian germplasms of Cucumis sativus and Cucumis melo." ISRN Agronomy. (2012). doi: 105402/2012/134134

https://www.hindawi.com/journals/isrn/2012/134 134.

\section{Cite this article as:}

Mala P., Payel D. and Sunita S. Molecular Phylogeny and Evolution of Tropical Vegetal Genus Coccinia (Cucurbitaceae) using Chloroplast barcode markers and its implications for speciation patterns. Annals of Plant Sciences 6.12 (2017) pp. 1817-1823.

doi: http://dx.doi.org/10.21746/aps.2017.6.12.3 